ISSN 1991-

website :http:// jsci.utq.edu.iq
الترقيم الدولي • 1991 - 199

Email: utjsci@utq.edu.iq

\title{
Off line Handwritten Signature Recognition based on Fusion of Global and GLCM Features Using Fuzzy Logic
}

\author{
Zamen F. Jabur \\ zamenfadhel@yahoo.com \\ Shaker K. Ali \\ shalynar@hotmail.com \\ University of Thi-Qar. College of Computers and Mathematics Sciences_Computers Department
}

\begin{abstract}
Signature is widely used and developed area of research for personal verification and authentication. In this paper, we present a new offline handwritten signature recognition system based on fusion of global and GLCM (Grey Level Co-occurrence Matrix) features using fuzzy logic system as classifier tool. The global and GLCM features are fused to generate vector of 15 features for the verification of the signature. The test signature is compared with the database signatures based on features, whilst match/non match of signatures is decided with fuzzy logic. The experimental results obtained by using a database of 7 individuals' signatures. A total number of 70 images are collected for our study and with average 10 signatures for each person, 5 of the signatures are used as training, the remaining 5 signatures are used as testing group. The results show that the proposed modular architecture can achieve $100 \%$ recognition accuracy for training group and $90.5 \%$ recognition accuracy for the testing group with running time is 1.17 second.
\end{abstract}

Key words: signature recognition, fuzzy logic, global features, GLCM features.

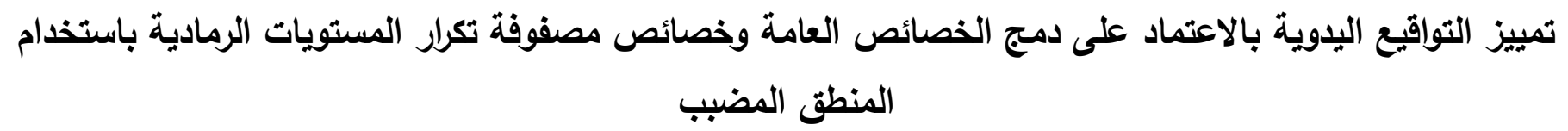

المستخلص

يستخدم التوقيع بصورة واسعة للتحقق من الأشخاص والتوثيق. في هذا البحث قدمنا نظام جديد لتمييز التواقيع اليدوية بالاعتماد على الخصائص العامة

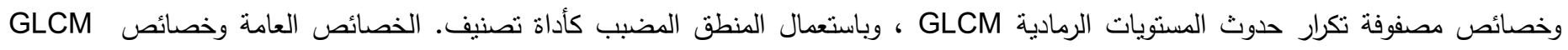

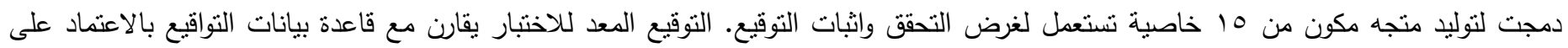

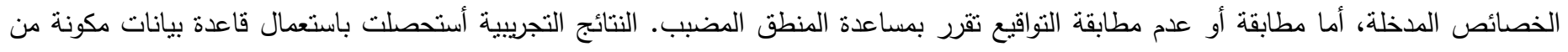

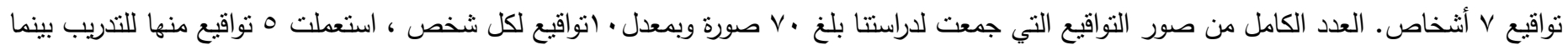

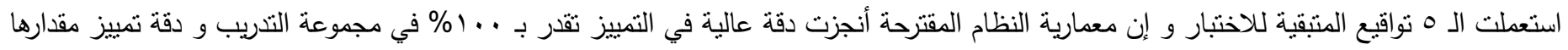
0, •9\% في مجموعة الاختبار وبمعدل زمن تتفيذ مقداره V V, أنانية.

\section{Introduction}

Biometrics is the science of automatic recognition of individual depending on their physiological and behavioral attributes. the expansion of networked society and increased use of some personal portable devices like tablet PCs, PDAs, mobile phones and authorization of access to sensitive data, is demanding the most reliable personal identification and authentication systems. Among the different forms of biometric recognition systems such as fingerprint, iris, face, voice, palm etc., signature will be most widely used. The applications like government and legal financial transaction, bank cheques use signature as one of personal identification system. The financial transactions and shopping using debit cards and credit cards require a bill to be confirmed by handwritten signature. But this leads to increased risk of financial loss to attempt forgeries. This problem may be resolved by introducing automatic recognition systems which are being successfully used effectively to analysis large quantities of biometric data [1].Overall, signature verification systems can be categorized as offline 
(static) and online (dynamic) [2]. Offline signature verification is obtained from a piece of writing paper which is scanned as an input image. It is mostly found on bank checks and documents signature. On the other hand, online signature verification uses the dynamic properties of the signature (signature trajectory, pen pressure, pen downs and pen ups, time stamping, etc) which are captured by a pen based tablet. Online signature verification is more robust, reliable, and accurate than offline signature verification as its dynamic properties make the process of forging an online signature more difficult [3]. However in the most common real-world scenarios, this information is not available, because it requires the observation and recording of the signing process. This is the main reason, why static signature analysis is still in focus of many researchers. Offline methods do not require special acquisition hardware, just a pen and a paper, they are therefore less invasive and more user friendly [4].It is supposed that the features of the process of signing originate from the intrinsic properties of human neuromuscular system which produces the aforementioned rapid movements. Knowing that this system is constituted by a very large number of neurons and muscle, fibers is possible to declare based on the central limit theorem that a rapid and habitual movement velocity profile tends toward a delta-log normal equation [5]. This statement explains the stability of the characteristics of the signature. Thus, the signature can be treated as an output of a system obscured in a certain time interval necessary to make the signature. This system models the person making the signature [6].This paper is organized as follows: In section 2, the Grey Level Co-occurrence Matrix (GLCM) is briefly introduced, in Section 3 our proposed method is presented it consists of read signature's image, preprocessing, feature extraction and classification stages. The experimental results are given in section 4 and finally conclusions are reported in section 5 .

\section{Grey Level Co-occurrence Matrix (GLCM)}

Haralicket all first introduced the use of cooccurrence probabilities using GLCM for extracting Gray various texture features. GLCM is also called as Gray level Dependency Matrix. It is defined as "A two dimensional histogram of gray levels for a pair of pixels, which are separated by a fixed spatial relationship." GLCM of an image is computed using a displacement vector $\mathrm{d}$, defined by its radius $\delta$ and orientation $\theta$ [7]. Haralick extracted thirteen texture features from GLCM for an image. we present important four features of these as follows $[8,9]$ :

2-1 Contrast: is a measure of intensity or gray-level variations between the reference pixel and its neighbor. In the visual perception of the real world, contrast is determined by the difference in the color and brightness of the object and other objects within the same field of view.

$$
\left.f_{1}=\sum_{n=0}^{N-1} n^{2}\left\{\sum_{i=0}^{N-1} \sum_{j=0}^{N-1} p_{d, 0}(i, j)\right)^{2}\right\}
$$

where:

$P_{d \cdot}$ gray level co-occurrence matrix.

d: displacement vector.

$\theta$ : the direction.

$(i, j)$ : of $P_{d}$ is the number of occurrences of the pair of gray levels $i$ and $j$ which the

distance between 1 and $\mathrm{j}$ following direction $\theta$ is $d$. $n=|i-j|$.

2-2 Correlation:feature shows the linear dependency of gray level values in the co-occurrence matrix. It presents how a reference pixel is related to its neighbor, 0 is uncorrelated, 1 is perfectly correlated.

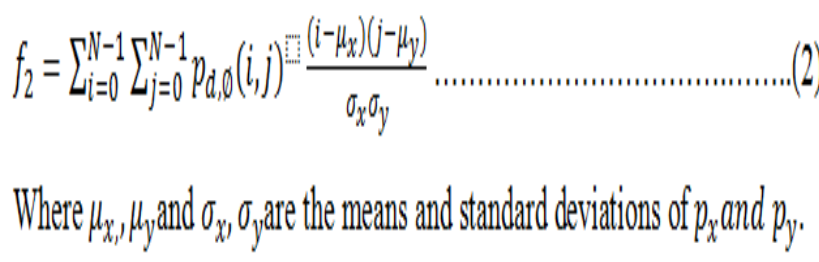

2-3 Energy: It measures the uniformity of an image. When pixels are very similar, the Angular Second Moment (ASM) also known as uniformity or energy, value will be large. 


$$
\begin{aligned}
& \left.f_{3}=\sum_{i=0}^{N-1} \sum_{j=0}^{N-1} p_{d, 0}(i, j)\right)^{2} \\
& \mu_{x}=\sum_{i=0}^{N-1} \sum_{j=0}^{N-1} i * p_{d, 0}(i, j) \mu_{y}=\sum_{i=0}^{N-1} \sum_{j=0}^{N-1} j * p_{d, 0}(i, j) \\
& \sigma_{x}=\sqrt{\left.\sum_{i=0}^{N-1} \sum_{j=0}^{N-1}(i-\mu)^{2} * p_{d, 0}(i, j)\right)} \sigma_{y}= \\
& \sqrt{\sum_{i=0}^{N-1} \sum_{j=0}^{N-1}(j-\mu)^{2} * p_{d, 0}(i, j)}
\end{aligned}
$$

2-4 Homogeneity: it is also known Inverse Difference Moment (IDM) that measures the local homogeneity of an image. IDM feature obtains the measures of the closeness of the distribution of the GLCM elements to the GLCM diagonal.

$$
f_{4}=\sum_{i=0}^{N-1} \sum_{j=0}^{N-1} \frac{1}{1+(i-j))^{2}} p_{d, 0}(i, j){ }^{-}
$$

Various research studies show $\delta$ values ranging from 1 , 2 to 10. Applying large displacement value to a fine texture would yield a GLCM that does not capture detailed textural information. From the previous studies, it has been concluded that overall classification accuracies with $\delta=1,2,4,8$ are acceptable with the best results for $\delta=1$ and 2. This conclusion is justified, as a pixel is more likely to be correlated to other closely located pixel than the one located far away. Also, displacement value equal to the size of the texture element improves classification.Every pixel has eight neighboring pixels allowing eight choices for $\theta$, which are $0^{\circ}, 45^{\circ}, 90^{\circ}, 135^{\circ}, 180^{\circ}, 225^{\circ}, 270^{\circ}$ or $315^{\circ}$. However, taking into consideration the definition of GLCM, the co-occurring pairs obtained by choosing $\theta$ equal to $0^{\circ}$ would be similar to those obtained by choosing $\theta$ equal to $180^{\circ}$. This concept extends to $45^{\circ}$, $90^{\circ}$ and $135^{\circ}$ as well. Hence, one has four choices to select the value of $\theta$. Sometimes, when the image is isotropic, or directional information is not required, one can obtain isotropic GLCM by integration over all angles [10].The dimension of a GLCM is determined by the maximum gray value of the pixel. Number of gray levels is an important factor in GLCM computation. More levels would mean more accurate extracted textural information, with increased computational costs. The computational complexity of GLCM method is highly sensitive to the number of gray levels and is proportional to quantized gray levels $(\mathrm{O}(\mathrm{G} 2))$ [10].

\section{3. proposed method}

The problem which we address in this paper is concerned with automatic recognition of person's signature that is captured on A4 paper. We have collected signatures from 7 different people which have unique personal signatures. Each person asked to sign 10 different samples on the paper then, this paper has been scanned with scanner to make our data set. Hence, a total number of 70 images are collected for our studies. The system is trained with 5 samples of each person's signatures and then it is tested by the 5 remaining. It means that a total number of 35 images are used to training and a number of 35 images for testing. The architecture schematic of our proposed system is shown in figure (1) where all details are clearly shown.

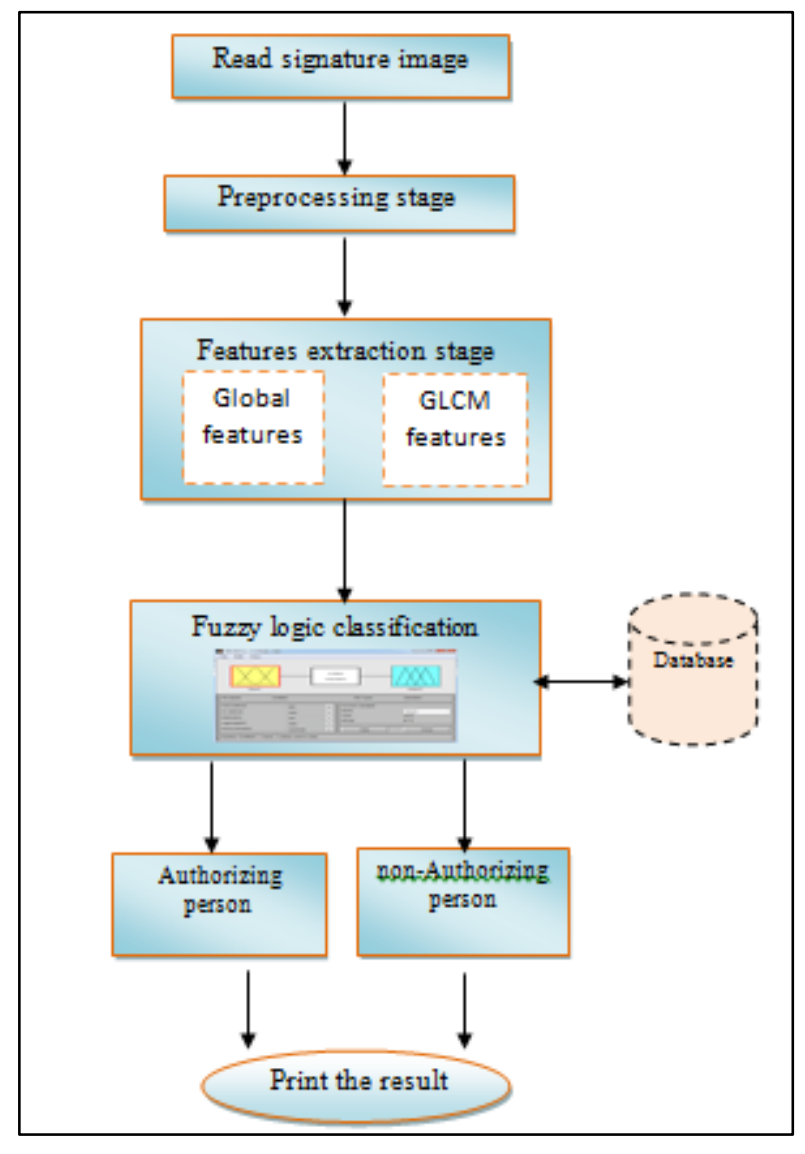

Figure (1) architecture schematic of the proposed svstem 


\section{3-1 Read signature image}

The signature image's entered to the system by using HP Scan Deskjet F2400 scanner with horizontal and vertical resolution $300 \mathrm{dpi}$, bit depth 24 , and the image store in JPEG format.

\section{3-2 Pre-processing stage:}

This stage consists of seven steps as it explain from section a to section $\mathrm{g}$ :

a) Convert color image to gray scale image:

A color image consists of coordinate matrix and three color matrix. Color matrices are known as Red $(\mathrm{R})$, Green $(\mathrm{G})$ and Blue (B). The designed system is based on gray scale images; therefore, colored image must be converted to gray scale using the equation bellow [11]:

$\mathrm{G}=0.299 * \operatorname{Red}+0.5876 *$ Green $+0-14 *$ Blue $\ldots$ (7)

\section{b) Image Thresholding:}

The goal of this step is to remove unnecessary information by using specific threshold so the image is converted to binary using the following equation:

Where $\mathrm{T}$ is desired threshold which obtained automatically using the following algorithm[12].

1. Select an initial estimate for $\mathrm{T}$ (midpoint between the minimum and maximum intensity value in the image.

2. Segment image using $\mathrm{T}$. This will produce two group of pixel G1consists from all pixel with gray level values $\geq T, G 2$ consists from all pixel with gray level values $<\mathrm{T}$.

3. Compute average gray level $m 1$ and $m 2$ for the pixels in the regions G1and G2.

4. Compute new threshold value:

$$
T=\frac{1}{2}(m 1+m 2)
$$

5. Repeat step 2 to step4 until difference in $\mathrm{T}$ in successive iteration smaller than predefined parameter $\mathrm{T}_{0}$.

Algorithm (1) global threshold method

\section{c) Remove Useless Object:}

In this step we search about connected component using 8-connected neighbors search in signature image and test it if it is size more than 5-pixel this consider useful object else if its size equal or less than 5-pixel we remove it by assign all pixels values to binary value zero.

\section{d) Image Trimming:}

The capture image may contain the signature and the area surrounding the signature which is empty of data. Thus, the signature must be separated from its background by tracing image from outside margins towards inside. Horizontal and vertical projection is used to calibrating the beginning and ending of signature to riddance from undesired areas of image.

\section{e) Size Normalization:}

Signature dimension may vary due to the scanning and capturing process. Furthermore, width and height of signature vary from person to person and sometimes even for the same person so that the image size is adjusted to $250 \times 250$ pixel.

\section{f) Image Closing:}

Often boundaries of signature images are quite ragged, the objects have false holes, and the background is peppered with small noise objects and holes founded in this objects. So morphological Closing can improve the situation markedly. In our proposed system we use morphological closing operation with disk shape structural element in size $3 \times 3$ in order to smooth the counter of signature image and filling the holes in it, figure (2) show signature image before and after apply morphological closing.
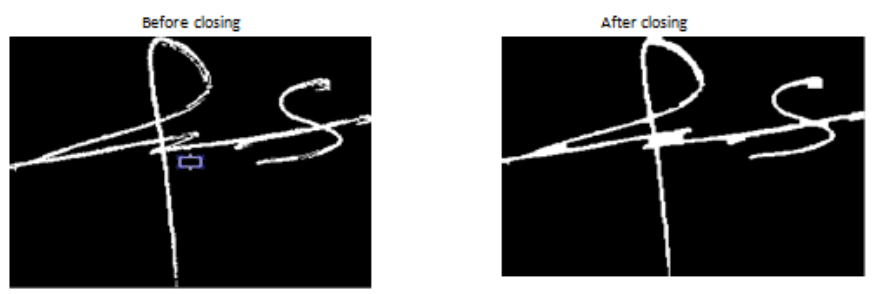

Figure (2) image before and after apply morphological closing

\section{g) Image Thinning:}

The goal of image thinning is to eliminate the width of signature from several pixels to single pixel, in order to signature backbone extraction which is considering 
common features in all entering signature, so that the object in resulted image has one pixel as shown in figure (3). Thinning makes the feature extraction more efficient.
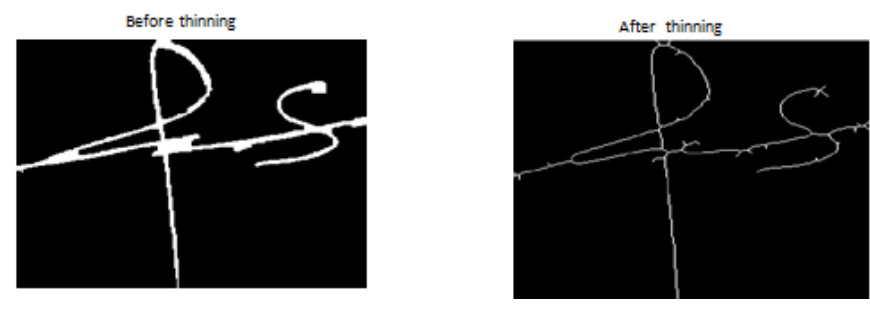

Figure (3) image before and after apply thinning

\section{3-3 Features extraction stage}

This stage converts each image into a set of features. In our system two groups of features (global features and GLCM features) are used in order to forming vector of 15 features, each person's signature image has its own vector features, these features are as follow:

\section{3-3-1 Global Features}

Provide information about specific cases concerning the structure of the signature. Global features contain 8 elements for each signature image which are:

a) Signature Height: The height of the signature can be considered as a way of representation, height-towidth ratio.

b) Image Area: Image area is the number of black pixels in the signature image.

c) Maximum Vertical Projection:The vertical projection of the thinning signature image is calculated by summing all the pixels along the columns. The vertical projection is define as [13]:

$$
V(j)=\sum_{i} I(i, j)
$$

\section{d) Maximum Horizontal Projection: The} horizontal projection of the thinning signature image is calculated by summing all black pixels along the rows, the horizontal projection is define as [13]:

$$
H(i)=\sum_{j} I(i, j)
$$

e) Vertical Center of the Signature: The vertical center of the signature Cy is given by [14]:

$$
C y=\frac{\sum_{y=1}^{y \max } y \sum_{x=1}^{x \max } b(x, y)}{\sum_{x=1}^{x \max } \sum_{y=1}^{y \max } b(x, y)}
$$

f) Horizontal Center of the Signature: The horizontal center $\mathrm{Cx}$ is given by [14]:

$$
C x=\frac{\sum_{x=1}^{x \max } x \sum_{y=1}^{y \max } b(x, y)}{\sum_{x=1}^{x \max } \sum_{y=1}^{y \max } b(x, y)}
$$

g) Number of Edge Point (En): An edge point is defined as a signature point that has only one 8neighbor as shown in figure (4) where it is symbols is $(\mathrm{E} 1, \mathrm{E} 2, \mathrm{E} 3, \mathrm{E} 4)$.

h) Number of Cross Point (Cn): Cross point is a signature point that has at least three 8neighbors as shown in figure (4) where it is symbols is $(\mathrm{C} 1, \mathrm{C} 2, \mathrm{C} 3, \mathrm{C} 4)$.

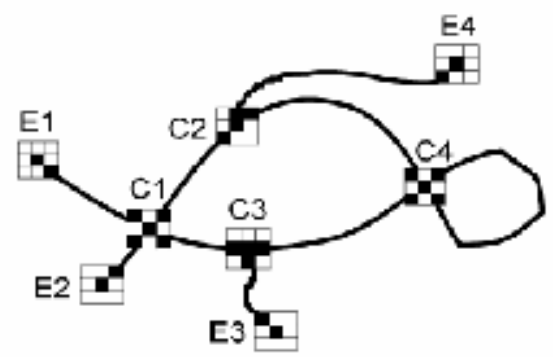

Figure (4) Edge point (E1,E2,E3,E4) and cross point $(\mathrm{C} 1, \mathrm{C} 2, \mathrm{C} 3, \mathrm{C} 4)$

\section{3-3-2 GLCM features}

Extract the features by using GLCM (Grey Level Co-occurrence Matrix) to extract the features that obtained from 16 features vector that is contain ( Homogenous, Energy, Contrast, correlation) in ( $0,45,90,135)$ degrees respectively.From this vector which contain 16 GLCM features we extract 7 major features which we are noticed it useful and distinct feature from image signature's person to other as following:

1- In degree 0 we extract contrast and correlation features.

2- In degree 45 we extract contrast and correlation features.

3- In degree 90 we extract correlation features only. 
4- In degree 135 we extract contrast and correlation features.

So that we get on vector consist from 15 feature 8 of them are resulted from global features and the remaining 7 features are resulted from GLCM features as shown in table (1) and table (2) which clear signature features vector for two person in training phase respectively and figure(5) and figure (6) show images for these signature's persons and curves for vector features of this signatures. This vector will enter to the next system stage (fuzzy logic) in order to classifier and verification.
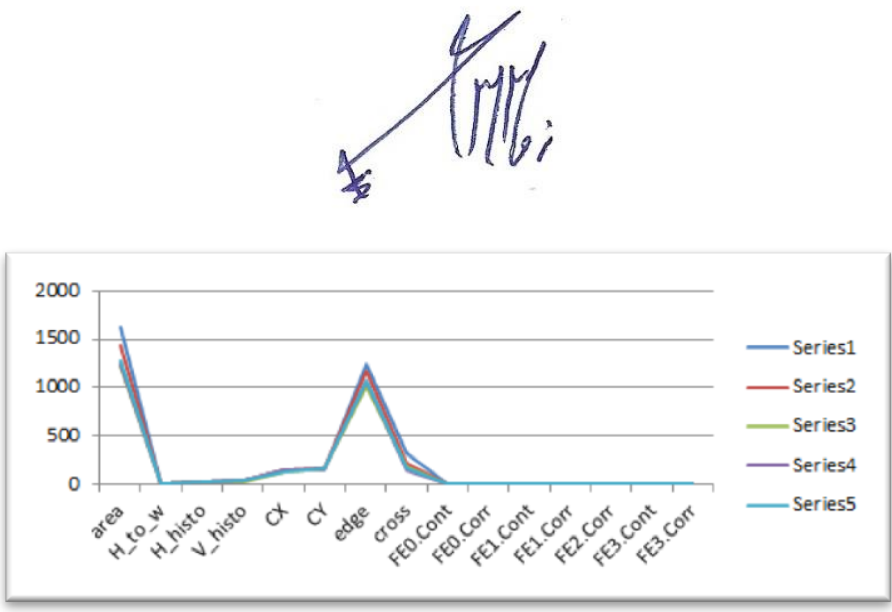

Figure (5) signature image's for one person and curve features

Table (1) five vector's features for five signatures belong to person in figure 5

\begin{tabular}{|c|c|c|c|c|c|c|c|c|c|c|c|c|c|c|c|}
\hline imgge & ares & $\begin{array}{l}\mathrm{B} 1 \mathrm{to} \\
\mathrm{II}\end{array}$ & \begin{tabular}{|c|} 
H \\
bisto \\
\end{tabular} & bisto & $a d$ & $\mathrm{CY}$ & ellge & cross & $\begin{array}{l}\text { FEO. } \\
\text { Cout }\end{array}$ & $\begin{array}{l}\text { FEO } \\
\text { Corr }\end{array}$ & $\begin{array}{l}\text { FEl. } \\
\text { Coost }\end{array}$ & $\begin{array}{l}\text { FLL. } \\
\text { Corr }\end{array}$ & $\begin{array}{l}\text { FLL. } \\
\text { Corn }\end{array}$ & $\begin{array}{l}\text { IFS. } \\
\text { Cout }\end{array}$ & $\begin{array}{l}\text { FES. } \\
\text { Corr }\end{array}$ \\
\hline 2l.jpg & 1021 & 0.67 & 19 & 30 & 146 & 141 & 1224 & 309 & 0.042 & 0.15 & 0.036 & 0.27 & 0.51 & 0.046 & 0.087 \\
\hline 12.jpg & 1422 & 0.63 & 17 & 28 & 130 & 161 & 1167 & 200 & 0.038 & 0.13 & 0.032 & 0.28 & 0.51 & 0.041 & 0.074 \\
\hline W.jpg & 1224 & 0.80 & 15 & 27 & 129 & 163 & 1006 & 176 & 0.033 & 0.13 & 0.027 & 0.28 & 0.51 & 0.036 & 0.078 \\
\hline 2d.jpg & 1236 & 0.70 & 15 & 36 & 134 & $1 \mathrm{~N}$ & 1068 & 134 & 0.033 & 0.12 & 0.028 & 0.17 & 0.52 & 0.036 & 0.008 \\
\hline ib.jpg & 1270 & 0.65 & 19 & 42 & 131 & 152 & 1058 & 160 & 0.034 & 0.12 & 0.028 & 0.28 & 0.50 & 0.036 & 0.075 \\
\hline range & \begin{tabular}{|l}
11244 \\
16211 \\
\end{tabular} & $\begin{array}{l}10.63- \\
0.081\end{array}$ & $\begin{array}{l}{[15} \\
19 \\
19\end{array}$ & $\begin{array}{l}{[27 \cdot} \\
42]\end{array}$ & $\begin{array}{l}{[129 .} \\
1146\end{array}$ & $\begin{array}{l}{[\mathrm{DA} \cdot} \\
1 \mathrm{~W}] \\
\end{array}$ & $\begin{array}{l}11006- \\
1234\end{array}$ & $\begin{array}{l}{[134 .} \\
309]\end{array}$ & $\begin{array}{l}{[0.035-1} \\
0.042]\end{array}$ & $\begin{array}{l}{[0.127 \%} \\
0.154]\end{array}$ & $\begin{array}{l}10.027 \\
0.0361\end{array}$ & $\begin{array}{l}{[0.274-} \\
0.2880\end{array}$ & $\begin{array}{l}10.507 \\
0.52222\end{array}$ & $\begin{array}{l}0.036 \\
0.046\end{array}$ & $\begin{array}{l}0.068- \\
0.0877\end{array}$ \\
\hline
\end{tabular}

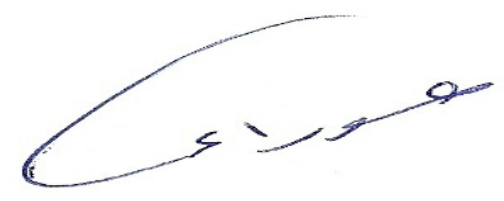

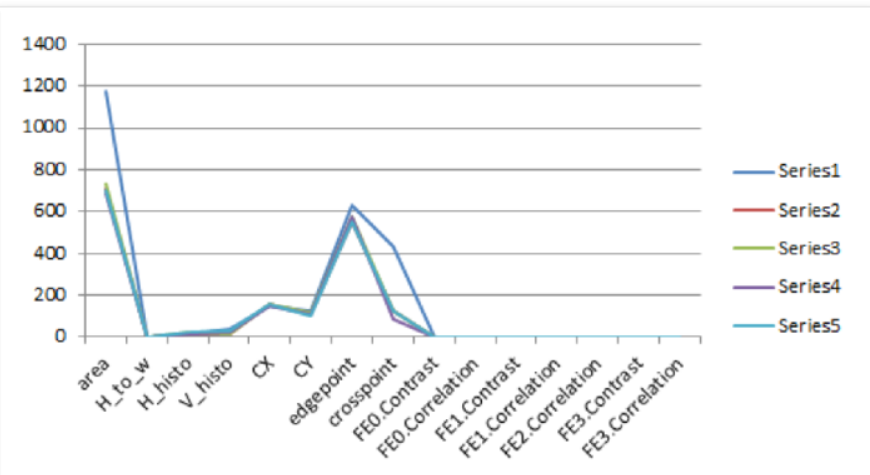

Figure (6) signature image's for one person and curve features

Table (2) five vector's features for five signatures belong to person in figure 6

\begin{tabular}{|c|c|c|c|c|c|c|c|c|c|c|c|c|c|c|c|}
\hline imagege & aree & $\begin{array}{c}10 \\
10 \\
\end{array}$ & \begin{tabular}{|c|}
$\mathrm{H}$ \\
bisto \\
\end{tabular} & \begin{tabular}{|c|}
1 \\
histo \\
\end{tabular} & $\alpha$ & $\mathrm{Cl}$ & ellge & cooss & $\begin{array}{l}\text { FEO } \\
\text { Cout } \\
\end{array}$ & $\begin{array}{l}\text { FEO. } \\
\text { Corr }\end{array}$ & $\begin{array}{l}\text { FIL } \\
\text { Cont }\end{array}$ & $\begin{array}{l}\text { FIL } \\
\text { Corn }\end{array}$ & $\begin{array}{l}\text { FE! } \\
\text { Corr }\end{array}$ & $\begin{array}{l}\text { FES. } \\
\text { Cont }\end{array}$ & $\begin{array}{l}\text { FES } \\
\text { Corn }\end{array}$ \\
\hline WL.jpg & 1171 & 0.67 & 15 & 36 & 150 & 124 & 626 & 4.4 & 0.026 & 0.17 & 0.027 & 0.26 & 0.42 & 0.020 & 0.20 \\
\hline W.jpg & 908 & 0.67 & 14 & 16 & 166 & 110 & 160 & 116 & 0.017 & 0.20 & 0.013 & $0.4 \mathrm{l}$ & 0.33 & 0.019 & 0.11 \\
\hline WS.jpg & 130 & 0.80 & 11 & 16 & 160 & 114 & 876 & 128 & 0.017 & 0.21 & 0.014 & 0.35 & 0.36 & 0.020 & 0.10 \\
\hline W.j.jpg & 688 & 0.85 & 14 & 4 & 116 & 107 & 876 & 80 & 0.017 & 0.20 & 0.011 & 0.4 & 0.30 & 0.019 & 0.08 \\
\hline Li.jpg & 698 & 0.82 & 20 & 11 & $1 \mathrm{~N}$ & 103 & \$ 19 & 121 & 0.018 & 0.17 & 0.013 & 0.37 & 0.10 & 0.19 & 0.09 \\
\hline rauge & $\begin{array}{l}\text { [688- } \\
1171]]\end{array}$ & $\begin{array}{l}0.67 \\
0.867\end{array}$ & {$\left[\begin{array}{l}{[14 .} \\
212]\end{array}\right.$} & $\begin{array}{l}166 . \\
360\end{array}$ & $\begin{array}{l}{[140 .} \\
1601\end{array}$ & $\begin{array}{l}{[103 .} \\
124]\end{array}$ & 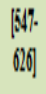 & 186. & $\begin{array}{l}{[0.017 \cdot} \\
0.026]\end{array}$ & $\begin{array}{l}{[0.17} \\
0.277\end{array}$ & $\begin{array}{l}{[0.011 .} \\
0.027]\end{array}$ & $\begin{array}{l}{[0.25} \\
0.4]\end{array}$ & $\begin{array}{l}{[0.30} \\
0.42]\end{array}$ & {$\left[\begin{array}{l}0.019 . \\
0.029]\end{array}\right.$} & $\begin{array}{l}10.08 . \\
0.201\end{array}$ \\
\hline
\end{tabular}

\section{3-4 Classification}

Fuzzy logic provides a simple way to arrive at a definite conclusion based upon vague, ambiguous, imprecise, noisy, or missing input information. Furthermore, fuzzy logic emulates how a person makes decisions and performs reasoning; however, fuzzy logic does it much faster $[15,16]$.There are many types of fuzzy neural network, for the forward multi-layer fuzzy neural network, the typical ones are Mamdani model, Sugeno model and Tsukamoto model. The simple and widely used Mamdani model is applied in this paper. For simplicity, as shown in figure (7) we assume the following rules in the fuzzy reasoning system of Mamdani model to know if the signature pictures authenticate or un-authenticate as following and:

$\mathrm{R} 1$ : If (input1 in [1224-1621]) and (input2 in [0.630.95]) and (input3 in [15-19]) and (input4 in [27-42] ) and (input5 in [129-145]) and (input6 in [141-154]) and (input7 in [1006-1234]) and (input8 in [134-309]) and 
(input9 in [0.033-0.042]) and (input10 in [0.1270.154]) and (input11 in [0.027-0.036]) and (input12 [0.274-0.285]) and (input13 in [0.507-0.522] ) and (input14 in [0.035-0.046]) and (input15 in [0.0680.087]) then (output1 is zamen) this person is authenticate.R2: If (input1 in [683-1171]) and (input2 in [0.67-0.85]) and (input3 in [14-22]) and (input4 in [16-36]) and (input5 in [145-160]) and (input6 in [103124]) and (input7 in [547-626]) and (input8 in [86434]) and (input9 in [0.017-0.026]) and (input10 in [0.17-0.27]) and (input11 in [0.011-0.027]) and (input12 [0.25-0.44]) and (input13 in[0.30-0.42]) and (input14 in [0.019-0.029]) and (input15 in [0.08-0.20]) then (output1 is hawraa) this person is authenticate.

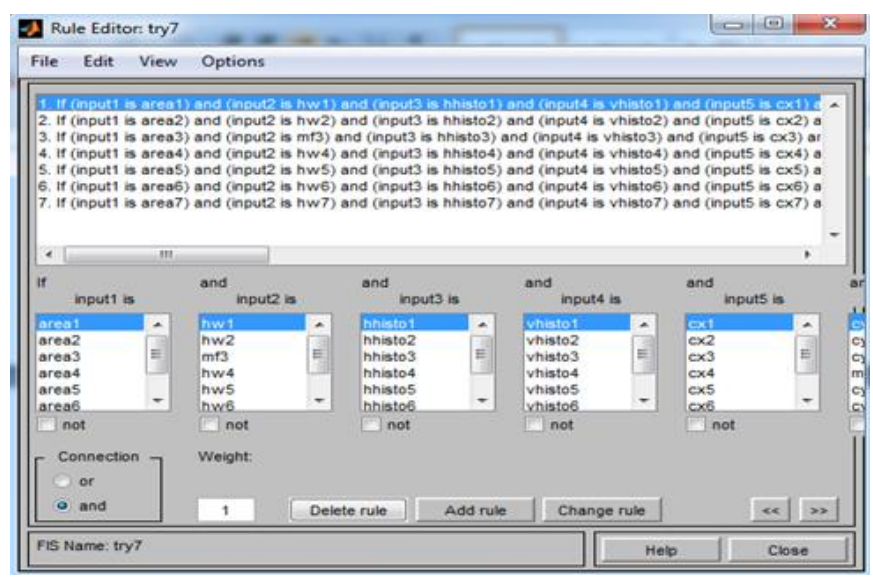

Figure (7) fuzzy logic rules for the proposed system

\section{4- Experimental Results}

Many experiments are performed to explore the possibility of the best parameters selection for handwritten signature recognition fuzzy logic classifier. Table (3) and table (4) show various experimental results of the multi-layer fuzzy neural network trained for the recognition of signatures for two persons. Our proposed system success in recognize the signature's person where we get on recognition rate is $100 \%$ in training phase and rate $90 \%$ in testing phase. Our system isn't depending on the pen or paper which the person makes his signature on it this gives our system robust and accuracy in determine the owner of signature.
Table (3) various experimental results of the multi-layer fuzzy neural network

\begin{tabular}{|c|c|c|c|c|c|c|c|c|}
\hline image/pers. & pers. 1 & pers. 2 & pers. 3 & pers. 4 & pers. .5 & pers. 6 & pers. 7 & the result \\
\hline zl.jpg & 15 & 6 & 7 & 7 & 3 & 3 & 4 & comect training \\
\hline 22.jpg & 15 & 5 & 7 & 7 & 1 & 5 & 4 & correct training \\
\hline 23.jpg & 15 & 7 & 8 & 9 & 6 & 3 & 4 & correct training \\
\hline 24.jpg & 15 & 5 & 8 & 7 & 7 & 3 & 4 & correct training \\
\hline 25.jpg & 15 & 4 & 7 & 10 & 5 & 4 & 5 & comect training \\
\hline 26.jpg & 11 & 8 & 5 & 9 & 5 & 2 & 3 & correct testing \\
\hline t7.jpg & 13 & 5 & 5 & 7 & 2 & 2 & 5 & correct testing \\
\hline 28.jpg & 9 & 5 & 6 & 7 & 4 & 3 & 5 & correct terting \\
\hline 20.jpg & 14 & 6 & 8 & 9 & 6 & 3 & 4 & correct terting \\
\hline z10.jpg & 12 & 5 & 5 & 8 & 4 & 3 & 2 & correct testing \\
\hline
\end{tabular}

Table (4) various experimental results of the multi-layer fuzzy neural network

\begin{tabular}{|c|c|c|c|c|c|c|c|c|}
\hline image/pers. & pers.1 & pers. 2 & pers. 3 & pers. 4 & pers. 5 & pers. 6 & pers.7 & the result \\
\hline bl.jpg & 4 & 15 & 7 & 8 & 5 & 4 & 6 & correct training \\
\hline h2.jpg & 2 & 15 & 5 & 4 & 5 & 6 & 4 & correct training \\
\hline h3.jpg & 1 & 15 & 6 & 6 & 5 & 6 & 6 & correct training \\
\hline h4.jpg & 4 & 15 & 6 & 4 & 4 & 5 & 2 & correct training \\
\hline b5.jpg & 3 & 15 & 7 & 7 & 5 & 7 & 6 & correct training \\
\hline 166.jpg & 3 & 13 & 8 & 10 & 3 & 7 & 7 & correct testing \\
\hline b7.jpg & 2 & 12 & 7 & 8 & 4 & 11 & 5 & correct testing \\
\hline h8.jpg & 3 & 14 & 6 & 7 & 3 & 7 & 5 & correct testing \\
\hline 49.jpg & 3 & 12 & 9 & 9 & 6 & 7 & 9 & correct testing \\
\hline h10.jpg & 3 & 13 & 10 & 9 & 6 & 7 & 9 & correct testing \\
\hline
\end{tabular}

\section{5- Conclusion}

The method described here is found to be successful in dealing with tilted and forged signature. The feature set formulated is found to be effective enough to capture finer variations in the signature. The work can be extended to include a wide class of signature and form an effective verification system. Our system isn't depend on type of imaging device where it success in recognize signatures are imaged using digital camera and it isn't depend on color of pen that is used to make the signature on paper.

\section{References}

[1]. Shashi Kumar et. al. "Off-line Signature Verification Based on Fusion of Grid and Global Features Using Neural Networks", International Journal of Engineering Science and Technology Vol. 2(12), 2010. 
[2]. Kholmatov and B. Yanikoglu. Identity authentication using improved on-line signature verification method. Pattern Recognition Letters, Volume 26, Issue 15, November 2005, pp. 24002408.

[3]. T.S. Ong, W.H. Khoh, A. Teoh. Dynamic handwritten signature verification based on statistical quantization Mechanism. IEEE Int Confon Computer Engineering and Technology, 2009.

[4]. B. Kovari, Z. Kertesz, and A. Major. Off-line signature verification based on feature matching. 11th IEEE Int Conf on Intelligent Engineering Systems, Budapest, Hungary, 29 June - 1 July 2007.

[5]. Plamondon, "The Handwritten Signature as a Biometric Identifier: Psychophysical Model \& System Design" IEE Conference Publications, R.1995,Issue CP408, 23-27.

[6]. Pacut, A. and Czaja,"Recognition of Human Signatures. Neural Network", A. 2001,in proceedings of the International Conference on Neural Network, IJCNN'01, vol.2, pp 15601564.

[7]. DhanashreeGadkari "Image Quality Analysis Using GLCM" thesis University of Central Florida Orlando, Florida,2004.

[8]. Sunita P. Aware "Image Retrieval Using CoOccurrence Matrix \&Texton Co-Occurrence Matrix For High Performance" International Journal of Advances in Engineering \& Technology, Vol. 5, Issue 2, pp. 280-291. 2013.

[9]. Tuan Anh Pham "Optimization of Texture Feature Extraction Algorithm" M.Sc thesis, Delft university, Netherlands, 2010.

[10]. Tuan AnhPham " Optimization of Texture Feature Extraction Algorithm" M.Sc.E thesis Delft University of Technology. 2010.

[11]. Cemil Oz, Fikret Ercal and Zafer Demir, "Signature Recognition and Verification with Artificial Neural Network Using Moment Invariant Method", Springer, Vol.3497, pp.195202, Berlin/Heidelberg, 2005.

[12]. R. C. Gonzalez, R. E. Woods, S. L. Eddins, "Digital Image Processing Using MATLAB(R)", Prentice Hall, 2004.

[13]. Scott Umbaugh, "Computer Vision and Image processing: A Practical Approach using CVIP Tools", Prentice-Hall, Inc., USA, 1998.

[14]. Emre Ozgunduz and M. Elif Karsligil, "Off-line Signature Verification and Recognition by
Support Vector Matching", Computer Engineering Department, Y1ld1z Technical University, Yıldız, Istanbul, Turkey, 2003.

[15]. Simone A. Ludwig, VenkatPulimi, AndriyHnativ "Fuzzy Approach for the Evaluation of Trust and Reputation of Services" International Conference on Fuzzy Systems, pp. 115-120, 2009.

[16]. Sukran YALPIR and Esra YEL "A Two Stage Fuzzy Model for Domestic Wastewater Treatment Plant Control" Water Observation and Information Systems conference BALWOIS 2010 . 